\title{
On Tomography with Unknown Orientation
}

\author{
Fawaz Hiouj \\ Department of Mathematics, The Petroleum Institute, United Arab Emirates \\ E-mail: fhiovi@pi.ac.ae
}

Received: August 23, 2015/ Accepted: November 9, 2015

\begin{abstract}
We consider the two-dimensional parallel beam Tomography problem in which both the object being imaged and the projection directions are unknown. The angles of projections need not to be uniformly distributed. Our solution combines two known approaches: the Geometric Moment and the Graph Laplacian. After sorting the projections using the Graph Laplacian method we create a one to one moment function of the angles. We then solve for each angle uniquely.
\end{abstract}

This is an Open Access article distributed under the terms of the Creative Commons Attribution License (http://creativecommons.org/licenses/by/3.0/), which permits unrestricted use, distribution, and reproduction in any medium, provided the original work is properly cited. 


\section{Introduction}

In this paper we consider the problem of two-dimensional parallel beam Tomography in which both the object and the projection directions are unknown. Projection directions may be unknown or known only approximately due to the uncontrollable motion of the object being imaged. For instance, this can happen in magnetic resonance imaging due to the motion of the patient.

Methods of recovering unknown view angles from the projection data have been proposed in [1, 2, 3, 4, and 5]. Of these, [4] and [5] utilize the concept of Geometric Moments of the projections. In [5], author relates the image's moments to the projections' moments then builds a nonlinear system of equations in which the angles of questions are among the unknown quantities of this system. Non uniqueness of solution in this approach was addressed by many authors such as in [4].In fact, [4] derives conditions for the existence of unique reconstruction from unknown directions and shifts. In addition, an algorithm for estimating the directions is introduced in [3], it consists three steps: 1) Initial direction estimation; 2) Direction ordering;3) Joint maximum likelihood refinement of the directions and shifts.

Another approach: [1] and [2] is fundamentally based on the principal component analysis (PCA) or the multi-dimensional scaling (MDS). It consists of constructing an $N \times N$ matrix whose entries are obtained from similarities between pairs of projections, followed by a computation of the first few eigenvectors of the similarity matrix. This approach builds on the fact that the map $\varphi \rightarrow f_{\varphi}^{\vee}$ is continuous from $\mathrm{S}^{1}$ to the space $\mathrm{L}_{2}(R)$; where $f_{\varphi}^{\vee}$ is the Radon Transform of the function

$f$ at angle $\varphi$, and $S^{1}$ is the unit circle. The Graph Laplacian tool was employed in [2] for sorting the given projections with respect to their directions where the ordering was obtained by a proper application of the diffusion map framework.

Our tools are the Geometric Moment and the Graph Laplacian combined. Indeed, we used the moments in dealing with transformation problems in [6] and [10].

Briefly speaking: After sorting the projections using Graph Laplacian method we work with the moments of the projections to create a one to one moment function of the angles. We then solve for each angle uniquely and accurately.

In so doing, [2] will be a major part of our algorithm. A brief description to this approach will be given shortly. However, readers who are not familiar with the application of the Graph Laplacian to Tomography may review [1] and [2]. 
In the remaining parts of this introduction we introduce notation and basic formulas. Following [7], let $f$ be a 2-D nonnegative function on the $\mathbf{x y}$ - plane with a compact support and $\xi=\left(\begin{array}{c}\cos \varphi \\ \sin \varphi\end{array}\right)$ is a unit vector; the Radon Transform of $f$ along the line $L=\{(x, y): x \cos \varphi+$ $y \sin \varphi=p\}$ is given by:

$f^{\vee}(p, \varphi)=f_{\varphi}^{\vee}(p)=\int_{-\infty}^{\infty} f(p \cos \varphi-t \sin \varphi, p \sin \varphi+t \cos \varphi) d t$,

We may use the vector form $f^{\vee}(p, \varphi)=f^{\vee}(p, \xi)$

For any $\varphi \in[0, \pi]$ and $k=0,1,2 \ldots$ we denote the $\mathrm{k}^{\text {th }}$ moment of the projection $f^{\vee}(p, \varphi)$ by:

$M f_{k, \varphi}=M f_{k}(\varphi):=\int_{-\infty}^{\infty} p^{k} f^{\vee}(p, \varphi) d p$,

We sometimes use the notation $M f_{k}(\varphi)$ to stress on the fact that $M f_{k}$ is a function of $\varphi$. However, $M f_{k, \varphi}$ will be used.

\section{Problem Formulation}

Let $f$ be an unknown nonnegative function with compact support on the $x y$-plane, for which we have $N$ projections, say $f^{\vee}\left(p, \varphi_{j}\right) ; j=1, \ldots, N$ at the unknown angles $\varphi_{j}$. We consider the problem of recovering the projection angles. However, the problem takes different forms due to different mathematical assumptions associated with it. Examples of variations of mathematical assumptions include: whether or not $N$ is large enough in the sense that we have continuum values of $f^{\vee}\left(p, \varphi_{j}\right)$. Or, whether a few number of projections (may be two or three) are already known, which is realistic in some applications. Or, whether the center of mass of $f$ need not to be on the origin, and so on.

In this paper, we consider the following problem:

\section{Problem:}

Let $f$ be nonnegative function on the $x y$-plane with support on the region $[0, a] \times[0, a]$ where $a$ is a positive number. Assume that we have $N$ projections $f^{\vee}\left(p, \varphi_{j}\right), j=1, \ldots N$ so that $\left\{\varphi_{j}\right\}$ is a fine sample of $[0, \pi]$ but not necessarily uniformly distributed. Our goal is to determine the angles of projections and reconstruct the unknown image $f$. 


\section{Review of Relevant Results}

We organize this section in two parts: first, we review some relevant basic results on the Geometric Moments. Second, we briefly describe the Graph Laplacian algorithm- as a faithful approach-used to recover the correct ordering of the projections.

\subsection{Review of Moments of Radon Projections}

Consider (2): we briefly review some basic relevant results regarding the $\mathbf{k}^{\text {th }}$ moment $M f_{k}(\varphi)$ of the projection $f^{\vee}(p, \varphi)$. It is well known, [8], that:

$$
M f_{k}(\varphi)=\sum_{j=0}^{k}\left(\begin{array}{l}
k \\
j
\end{array}\right) \cos ^{k-j}(\varphi) \cdot \sin ^{\mathrm{j}}(\varphi) . \quad \iint_{-\infty-\infty}^{\infty} x^{k-j} y^{j} f(x, y) d x d y
$$

That can be written as:

$M f_{k}(\varphi)=\mu_{k, 0} \cos ^{k} \varphi+\mu_{k-1,1} \cos ^{k-1} \varphi \sin \varphi+\cdots+\mu_{0 k} \sin ^{k} \varphi$,

We choose to determine the first moment function using the angles

$\varphi=0$, and $\frac{\pi}{2}$. With direct calculations on (3) we may write:

$$
\begin{array}{r}
M f_{1}(\varphi):=\int_{-\infty}^{\infty} p f^{\vee}(p, \varphi) d p=M f_{1,0} \cos \varphi+M f_{1 \frac{\pi}{2}} \sin \varphi \\
=R \cos (\varphi+\alpha)
\end{array}
$$

\subsection{Review of the Graph Laplacian Approach}

A corner stone for applying the Graph Laplacian tool to our problem is the following proposition that was mentioned in [9] and proved in [1].

\section{Proposition 1:}

Suppose $f \in L_{2}\left(R^{2}\right)$ and that $f$ vanishes outside the unit disk.

Then, $f_{\varphi}^{\vee}$ is in $L_{2}(R)$ for all $\varphi$ and $\left\|f_{\varphi_{1}}^{\vee}-f_{\varphi_{2}}^{\vee}\right\|$ tends to zero as $\varphi_{1}$ approaches $\varphi_{2}$. In other words, the map $\varphi \rightarrow f_{\varphi}^{\vee}$ is continuous from $S^{1}$ to $L_{2}(R)$, where $S^{1}$ is the unit circle.

The measurements are assumed to be discrete samples of the Radon Transform $f^{\vee}(p, \varphi)$ with fine uniformly spaced values of the argument $p$ given at $p_{1}, p_{2}, \ldots p_{m}$. Every projection (vector), $f^{\vee}(p, \varphi)$, is viewed as a point in $R^{m}$. When varying the beaming direction $\varphi$ over $S^{1}$, the projection vectors sample a parametric closed curve: $C$ in $R^{m}$ parametrized by $\varphi$. 
As said in the introduction, a major step of our proposed solution will require sorting the data with the assumptions that the beaming directions are not necessarily uniformly distributed over $S^{1}$ and that the projections (points) are not uniformly distributed over C.

In so doing, we will follow the approach of [2] whose method consists of constructing an $N \times N$ weight matrix whose entries are obtained from similarities between pairs of projections, followed by a computation of the first few eigenvectors of the similarity matrix.

The fact that the projections are not uniformly distributed over $C$ suggest that we use the Density Invariant Graph Laplacian explained in [2].

This normalization leads to the Laplace-Beltrami operator, and the computed eigenvectors will be discrete approximations of the eigenfunctions of the Laplace-Beltrami operator over C.Specific usage and implementation of this algorithm is illustrated in section 5 of this paper.

\section{Proposed Approach}

To solve the stated problem, we divide the analyses in two parts: first we introduce our theoretical solution that combines the theory of Geometric Moments and the Graph Laplacian algorithm. Second, we adjust the analysis to fit the discrete setting of the problem.

\subsection{A Theoretical Solution}

Consider the moment function in (4): $M f_{1}(\varphi)=R \cos (\varphi+\alpha)$ on $[0, \pi]$

that is drawn in figure 1. Clearly, $R=\max M f_{1}(\varphi)$ on $[0, \pi]$.

Let $r:=\min M f_{1}(\varphi)$ on $[0, \pi]$. Observe that $r=R \cos (\pi+\alpha)$, equivalently:

$\alpha=-\cos ^{-1}\left(\frac{-r}{R}\right)$

$\alpha<0$ and $0 \leq|\alpha| \leq \frac{\pi}{2}$

Observe also that $M_{0}:=R \cos (\alpha)>0$.

Notice that $M f_{1}(\varphi)$ is not an one to one function on $[0, \pi]$. Thus, for an individual projection $f^{\vee}(p, \varphi)$ with unknown $\varphi \in[0, \pi]$, the equation

$M f_{1}(\varphi)=\operatorname{Rcos}(\varphi+\alpha)$ has two possible solutions in $[0, \pi]$. Clearly, more work is needed to determine the correct angle.

Extend $M f_{1}(\varphi)$ using the fact that

$f^{\vee}(p, \varphi+\pi)=f^{\vee}(-p, \varphi)$, 
So we have $M f_{1}(\varphi)$ on $[0,2 \pi]$. In this way, $f^{\vee}(p,|\alpha|)$ and $f^{\vee}(p, \pi-\alpha)$ are the two projections of maximum and minimum moments, $R$ and $-R$ respectively.

Let $C$ be the continuous simple closed parametric curve in $R^{m}$ parameterized by $\varphi \in$ $[0,2 \pi]$. Each point on $C$ is the Radon Transform $f^{\vee}(p, \varphi)$ with a fine uniformly spaced values of the argument $p$ given at $p_{1}, p_{2}, \ldots p_{m}$.

In particular, let $\mathcal{P}_{1}$ and $\mathcal{P}_{2}$ be the two points on $\mathrm{C}$ that represent the projections $f^{\vee}(p,|\alpha|)$ and $f^{\vee}(p, \pi-\alpha)$ respectively. Then, there are only two ways

(Orientations) to travel on $\left(\right.$ from $\mathcal{P}_{1}$ to $\mathcal{P}_{2}$. For example, in figure 1, the part of the graph on $[|\alpha|, \pi-\alpha]$ can be (identified) or represented by the function:

$$
M f_{1}(\varphi)=R \cos \left(\theta_{\varphi}\right) ; \theta_{\varphi} \in[0, \pi] \text { and } \varphi \in[|\alpha|, \pi-\alpha]
$$

Solving (8) for $\theta_{\varphi}$ we obtain:

$\theta_{\varphi}=\cos ^{-1}\left[\frac{M f_{1}(\varphi)}{R}\right]$

However, due to the Rotation and Reflection Rules of Radon Transform we may eventually producing an $\alpha$-rotated image, or reflected image rather than the original one.

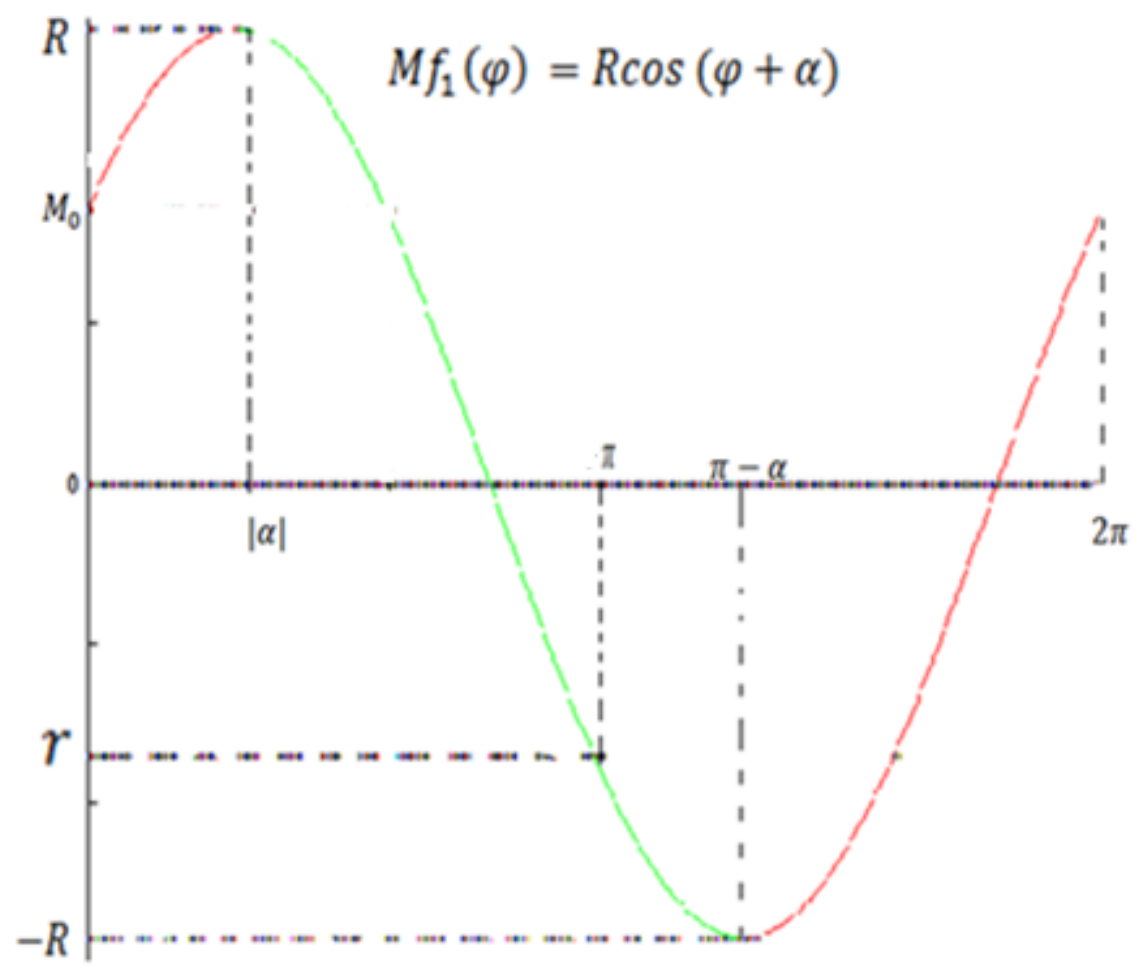

Figure1. The shifted cosine $M f_{1}(\varphi)$ on $[0,2 \pi]$ showing the factors $R, r, \alpha$, and $M_{0}$ 


\subsection{The Discrete Setting of the Problem}

Given $N$ projections $f^{\vee}\left(p, \varphi_{j}\right), j=1, \ldots N$ so that $\varphi_{j}$ is a fine sample of $[0, \pi]$.

We assume that we have fine uniformly spaced values of the argument $p$ given at $p_{1}, p_{2}, \ldots p_{m}$. We double the available data using the fact that $f^{\vee}\left(p, \varphi_{j}+\pi\right)=f^{\vee}\left(-p, \varphi_{j}\right)$ so we have $2 N$ projections on $[0,2 \pi]$. We store these data as columns of the $m \times 2 N$ matrix $\mathcal{R}_{0}$.

Now, we subject the columns of $\mathcal{R}_{0}$ to the Graph Laplacian algorithm; more details are presented in the next section.

Let $\mathcal{R}$ be the $m \times 2 N$ matrix of the ordered projections

Let $\mathcal{M}$ let be the vector of moments of the columns of $\mathcal{R}$ such that

$\mathcal{M}_{j}=M f_{1}\left(\varphi_{j}\right), j=1 \ldots, 2 N$

Consider the function $M f_{1}(\varphi)=R \cos (\varphi+\alpha)$ : we observe that

$R \approx \max \mathcal{M}$. Indeed, knowing $\alpha$ is not critical to our proposed calculations, however, it can be approximated using (6).

Let $j_{1}$ and $j_{2}$ be the locations of $f^{\vee}(p,|\alpha|)$ and $f^{\vee}(p, \pi-\alpha)$ in $\mathcal{R}$.

These are the projections correspond to the points $\mathcal{P}_{1}$ and $\mathcal{P}_{2}$ described above.

Thus, we can work with the set of projections $j_{1}, \ldots, j_{2}$ individually and for each projection of these, say $f^{\vee}\left(p, \varphi_{j}\right)$, we have:

$\theta_{j}=\cos ^{-1}\left[\frac{M f_{1\left(\varphi_{j}\right)}}{R}\right]=\cos ^{-1}\left[\frac{\mathcal{M} C_{j}}{R}\right]$

In this way, we obtain a sufficient set of projections with exact values of their angles $\theta_{j} \epsilon[0, \pi]$.

\section{Calculations and Examples}

We now summarize our approach and show some experiments.

\subsection{Algorithm}

First, we can summarize section 4.2 using the following algorithm:

1. Start with the $m \times 2 N$ matrix $\mathcal{R}_{0}$

2. Order $\mathcal{R}_{0}$ and get $\mathcal{R}_{\text {; }}$ see below

3. Compute $\mathcal{M}$ as in (10), $R \approx \max \mathcal{M}$, and identify $j_{1}$ and $j_{2}$

4. For each projection $f^{\vee}\left(p, \varphi_{j}\right)$ with $j_{1} \leq j \leq j_{2}$ compute $\theta_{j}=\cos ^{-1}\left[\frac{\mathcal{M}_{j}}{R}\right]$

5. Reconstruct the image 
For step 2: Given $f^{\vee}\left(p, \varphi_{j}\right) ; j=1, \ldots, N$. Using [2], we compute the 2-norm distances and the weight matrix $\mathrm{W}$ :

$\delta_{i j}=\left\|f^{\vee}\left(p, \varphi_{i}\right)-f^{\vee}\left(p, \varphi_{j}\right)\right\|$

$W_{i j}=\exp \left[\frac{-\delta_{i j}{ }^{2}}{2 \epsilon}\right], i, j=1, \ldots, N, \epsilon>0$

Normalize $\quad W$ using the diagonal matrix $D$ with $D_{i i}=\sum_{j=1}^{n} W_{i j}$, and form the Graph Laplacian $L=D^{-1} W-I$ where $I$ is $N \times N$ identity matrix.

Next, we obtain the Density Invariant Graph Laplacian $L_{1}$ by:

$L_{1}=D_{1}^{-1} W_{1}-I$,

With $W_{1}=D^{-1} W D^{-1}$ and $D_{1}$ is the diagonal matrix: $\left(D_{1}\right)_{i i}=\sum_{j=1}^{N} W_{1_{i j}}$

Then, we compute $v_{1}$ and $v_{2}$, the first nontrivial eigenvectors of $L_{1}$, then use the diffusion map to sort the projections $f^{\vee}\left(p, \varphi_{j}\right)$ according to

$\omega_{j}=\tan ^{-1}\left[\frac{v_{1}(j)}{v_{2}(j)}\right]$

\subsection{Examples}

We show two of our experiments: in the first example, we consider a block image from a family of images that we developed in [6]. This family provides good phantoms and efficient numerical tools for carrying calculations such as the Radon Transform or the moment's integrals. The Block $F$ shown in figure $2 a$ is subjected to Algorithm 5.1 with beaming direction is not uniformly distributed as in figure 2 and is uniformly distributed as in figure 3 . In both cases, the algorithm works fine and recover the exact angles of projections. We repeated the experiments on a brain image as in figures 4 and 5 . Clearly, in all of these outputs: images reconstructed from uniformly beaming directions are smoother than the ones that are not. But this is not the concern in this paper because what matters in these experiments is to show that we can recover the angles of projections in all cases. 


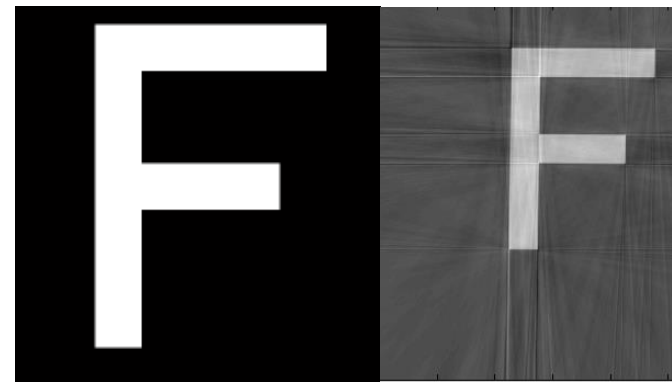

(a) (b)

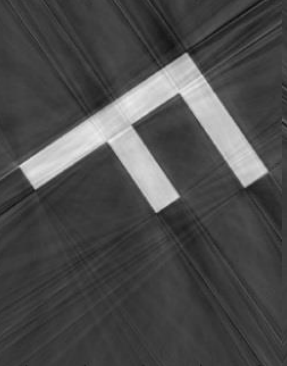

(c)

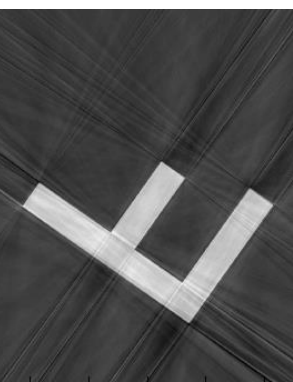

(d)

Figure2. Beaming direction is not uniformly distributed. In MATLAB: Angle = (pi.*rand(300,1))' a). the original image. b). recovered image using iradon assuming angles of projections are known. c and d). Recovering using the two possible orientations from $\mathrm{j}_{1}$ to $\mathrm{j}_{2}$ as in algorithm 5.1.

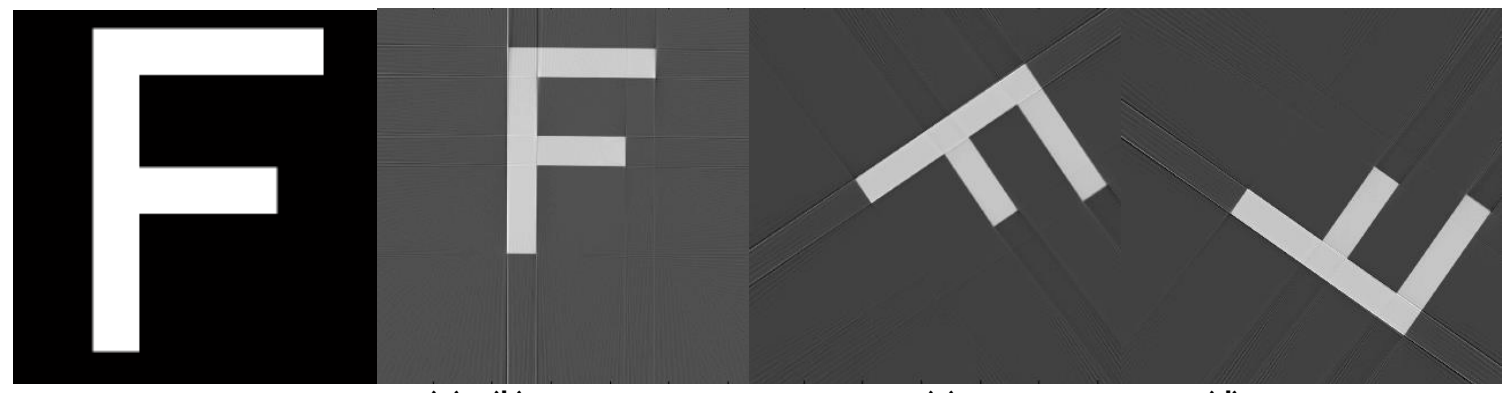

(a) (b)

(c)

(d)

Figure3. Beaming direction is uniformly distributed. In MATLAB: Angle=linspace (0,pi,300). a). the original image. b). recovered image using iradon assuming angles of projections are known.

c and d). Recovering using the two possible orientations from $\mathrm{j}_{1}$ to $\mathrm{j}_{2}$ as in algorithm 5.1.

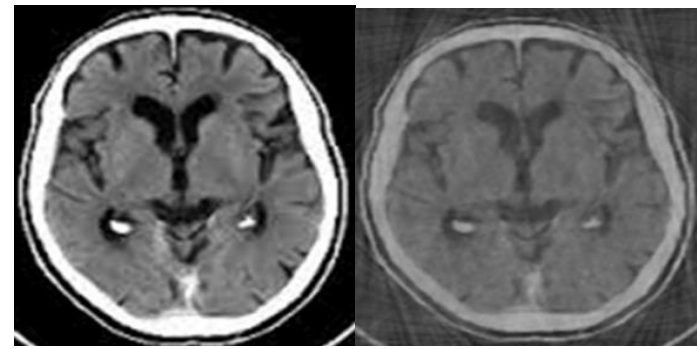

(a)(b)

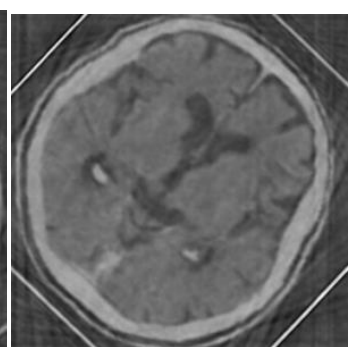

(c) (d)

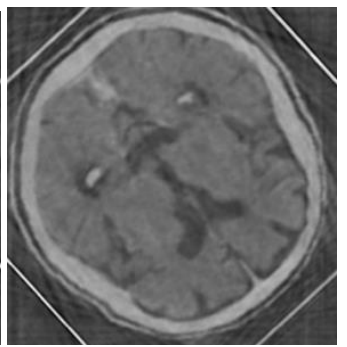

Figure4. Beaming direction is not uniformly distributed. In MATLAB: Angle $=\left(\text { pi. }{ }^{*} \text { rand }(300,1)\right)^{\prime}$ a). the original image. b). recovered image using iradon assuming angles of projections are known.

c and d). Recovering using the two possible orientations from $j_{1}$ to $j_{2}$ as in algorithm 5.1.

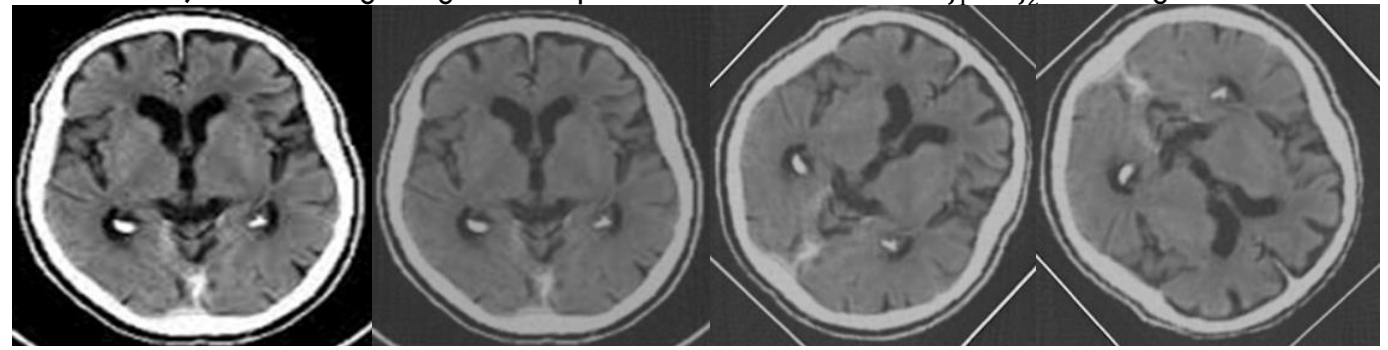

(a) (b)

(c)

(d)

Figure5. Beaming direction is uniformly distributed. In MATLAB: Angle=linspace $(0, p i, 300)$. a). the original image. b). recovered image using iradon assuming angles of projections are known.

c and d). Recovering using the two possible orientations from $j_{1}$ to $j_{2}$ as in algorithm 5.1 


\section{Conclusion}

We have reviewed the problem of Tomographic reconstruction when the orientation (angles of projections) are unknown and need not to be uniformly distributed over the unit circle. In doing so, we have combined the Moment and the Graph Laplacian approaches. It is possible to obtain two possible angles for each projection using Geometric Moments of the projections. However, this can't determine the correct angle.

We formed a simple closed parametric curve $C$ in $R^{m}$ parameterized by the angles of projections $\varphi \in[0,2 \pi]$. Each point on that curve is the Radon Transform $f^{\vee}(p, \varphi)$ with fine uniformly spaced values of the argument $p$ given at $p_{1}, p_{2}, \ldots p_{m}$. We used the Graph Laplacian approach as a known tool for sorting the projections according to their angle of projections. Two particular points $\mathcal{P}_{1}$ and $\mathcal{P}_{2}$ on $C$ are of critical importance. These points correspond to the projections with maximum and minimum moments. There are only two ways (Orientations) to travel on $\mathrm{C}$ from $\mathcal{P}_{1}$ to $\mathcal{P}_{2}$. One way produces a rotated image and the other produce a rotatedreflected image. Indeed, we can calculate the angle of rotation. We tested several types of images and we show the results of our algorithm on a block image as well as a brain image. Algorithm works and recover the exact angles whether or not the beaming directions are uniformly distributed over $S^{1}$.

Our approach requires a fine sample of projections, same as [2]. However, [2] assumes the projection angles to be uniformly distributed over the circle so that they can be estimated after recovering the correct ordering of the projections.

A related problem in the literature is to determine the angles of projections when only a few projections are available as in [5] that solved the problem but did not address the uniqueness of its proposed solution. Indeed, the fact that we can completely determine $M f_{1}(\varphi)$ in (4) using only two orthogonal projections is encouraging observation, that is because for an individual projection $f^{\vee}(p, \varphi)$ with unknown $\varphi \in[0, \pi]$ we always can use $M f_{1}(\varphi)$ and obtain (only) two possible solutions. A proper use of higher order moments of the projections or some other types of moments may improve the current approaches. We will be researching these possibilities in a future work.

\section{Acknowledgements}

The author thanks the Petroleum Institute for the support through a 2015 A\&S Research Grant. 


\section{References}

1. Singer $A$ and $H$ Wu (2013) Two-dimensional tomography from noisy projections taken at unknown randomdirections. SIAM J Imaging Sc. 6 (1): 136-175.

2. Coifman RR, Y Shkolnisky, FJ Sigworth, and A Singer (2008) Graphlaplaciantomography from unknown random projections. IEEE Trans. Image Proc. 17 (10): 1891-1899.

3. Basu S and Y Bresler (2000) Feasibility of tomography with unknown view angles. IEEE Transactionson Image Processing 9 (6): $1107-1122$.

4. Basu $S$ and $Y$ Bresler. Uniqueness of tomography with unknown view angles. IEEE Transactionson Image Processing 9 (6): 1094-1 106.

5. Goncharov A (1990) Mathematical Problems of Tomography (Translations of Mathematical Monographs Vol 81. Ed Gelfand I and Gindikin S (Providence, Rhode Island: American Mathematical Society), pp. 67-96. (Translated from the Russian by S. Gelfand).

6. Hjouj F, and DW Kammler (2008) Identification of reflected, scaled, translated, and rotated objects from their Radon projections. IEEE Trans Image Process 17 (3): 301-310.

7. Deans SR (1983) The Radon Transform and Some of Its Applications, New York. John Wiley \& Sons, Inc.

8. Milanfar P, WC Karl, and AS Willsky (1999) Recovering the Moments of a Function From Its Radon-Transform Projections: Necessary and Sufficient. Laboratory for Information and Decision Systems. Massachusetts Institute of Technology Cambridge, Massachusetts, USA.

9. Epstein CL (2003) Introduction to the mathematics of medical imaging. Pearson Education, Upper Saddle River, New Jersey, USA.

10. Hjouj F (2011) Linear Transformation Recognition Using Radon Transform. J Math Sci \& Computer Appl 1 (2): 40-47. doi: 10.5147/jmsca.2011.0038. 